\title{
Rhubarb extract has a protective role against radiation-induced brain injury and neuronal cell apoptosis
}

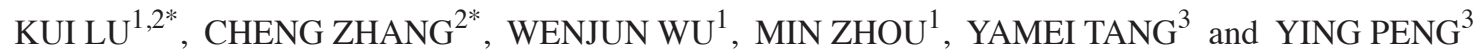 \\ ${ }^{1}$ Department of Neurology, Zhongshan City People's Hospital, Zhongshan, Guangdong 528403; ${ }^{2}$ Department of Neurology, \\ First Affiliated Hospital of Sun Yat-sen University, Guangzhou, Guangdong 510080; ${ }^{3}$ Department of Neurology, \\ Sun Yat-sen Memorial Hospital of Sun Yat-sen University, Guangzhou, Guangdong 510120, P.R. China
}

Received January 16, 2014; Accepted August 14, 2014

DOI: $10.3892 / \mathrm{mmr} .2015 .3693$

\begin{abstract}
Oxidative stress caused by ionizing radiation is involved in neuronal damage in a number of disorders, including trauma, stroke, Alzheimer's disease and amyotrophic lateral sclerosis. Ionizing radiation can lead to the formation of free radicals, which cause neuronal apoptosis and have important roles in the development of some types of chronic brain disease. The present study evaluated the effects of varying concentrations $(2,5$ and $10 \mu \mathrm{g} / \mathrm{ml})$ of ethanolic rhubarb extract on the neuronal damage caused by irradiation in primary neuronal cultures obtained from the cortices of rat embryos aged 20 days. Brain damage was induced with a single dose of $\gamma$-irradiation that induced DNA fragmentation, increased lactate dehydrogenase release in neuronal cells and acted as a trigger for microglial cell proliferation. Treatment with rhubarb extract significantly decreased radiation-induced lactate dehydrogenase release and DNA fragmentation, which are important in the process of cell apoptosis. The rhubarb extract exhibited dose-dependent inhibition of lactate dehydrogenase release and neuronal cell apoptosis that were induced by the administration of ionizing radiation. The effect of a $10 \mu \mathrm{g} / \mathrm{ml}$ dose of rhubarb extract on the generation of reactive oxygen species (ROS) induced by radiation was also investigated. This dose led to significant inhibition of ROS generation. In conclusion, the present study showed a protective role of rhubarb extract against irradiation-induced apoptotic neuronal cell death and ROS generation.
\end{abstract}

Correspondence to: Dr Kui Lu, Department of Neurology, Zhongshan City People's Hospital, 2 Sunwen East Road, Zhongshan, Guangdong 528403, P.R. China

E-mail: kuilu999@yahoo.com; lukui945@163.com

${ }^{*}$ Contributed equally

Key words: brain-injury, apoptosis, rhubarb, reactive oxygen species, lactate dehydrogenase

\section{Introduction}

An estimated 18,000 new cases of brain and central nervous system tumors are diagnosed annually, and $\sim 13,000$ people will succumb to their disease in the United States (1). Despite the availability of the latest neuroimaging techniques and advances in treating primary tumors, cases of brain cancer continue to rise. The primary treatment option for these patients is complete or partial brain irradiation. Radiotherapy is a well-established modality for the treatment of a number of types of cancer. It is estimated that approximately half of the patients with brain cancer receive radiotherapy as part of a treatment strategy. Each year, 200,000 patients with brain cancer are treated with partial or whole brain irradiation. However, the therapeutic effects are limited by the harmful consequences of the post-irradiation injuries sustained by healthy normal cells (2-5). In the case of brain cancer irradiation, these injuries may give rise to irreversible cognitive impairment, which is accompanied by an increase in mortality and morbidity. This cognitive impairment is hypothesized to be a result of the oxidative stress that occurs as a result of irradiation. The free radicals present are predominantly reactive oxygen species (ROS) that are generated as a result of ionizing radiation leading to DNA destruction, such as single or double-strand breaks, base damage and DNA-DNA or DNA-protein cross-links. The DNA double strand breaks are hypothesized to be the most damaging events that occur following the administration of ionizing radiation, and have been found to be the principal mechanism of irradiation-induced cell death. It has also been reported that DNA damage due to irradiation leads to apoptosis. Apoptosis is an important mechanism of neuronal cell death in rapidly and slowly progressive brain diseases. Experimental evidence suggests that radiation triggers the formation of microglial cocultures as well as astrocytes in vitro, elevating the expression of cyclooxygenase-2 (COX-2), interleukin (IL)- $1 \beta$, IL-6 and tumor necrosis factor (TNF)- $\alpha$, which are key proinflammatory mediators (6-13).

There are currently no effective, evidence-based treatments available for radiation-induced brain injury leading to cognitive impairment and other brain diseases. A number of botanical extracts have been investigated in this context. Examples include the different extracts of 
Ginkgo biloba, Centella asiatica, Hippophae rhamnoides, Osimum sanctum, Panax ginseng, Podophyllum hexandrum, Tinospora cordifolia, Piper longum, Mentha arvensis and Mentha piperita. Numerous natural and herbal products, which are of medicinal use have become important ingredients in the human diet. The capacity of dietary ingredients to provide protection against radiation-induced injury has until now remained unexplored. Radioprotective dietary supplements may be an ideal form of treatment, as they are used frequently as part of a normal diet, as they are non-toxic and have minimal side effects (14).

Rheum officinale Baill. (a member of the Polygonaceae family) is a perennial herb, the dried roots and rhizomes of which are commonly termed rhubarb. Rheum officinale is also called Chinese rhubarb and is used in traditional Chinese medicine, where it is termed 'Yao yong dahuang'. Anthraquinones, dianthraquinones, stilbenes, flavonoids and polyphenols are the principal phytoconstituents of the majority of rhubarbs. Emodin (1,3,8-trihydroxy-6-methylanthraquinone) is a naturally occurring anthraquinone present in the roots and rhizomes of Chinese herbs, including Rheum officinale and Rheum emodi. Rhubarb has been reported to have several pharmacological effects, including anti-inflammatory, antibacterial, purgative and anticancer properties. Certain species of Rheum have been used against radiation-induced immune damage in rats (15-17).

The aim of the present study was to evaluate the radioprotective action of Rheum officinale extract against neuronal apoptotic cell death and ROS generation induced by ionizing radiation. Phytochemical analysis by liquid chromatography-mass spectrometry (LC-MS) and high performance liquid chromatography (HPLC) were also conducted, which led to the identification of five chemical constituents that were present in the extract.

\section{Materials and methods}

Materials. Bis-benzimide was obtained from Sigma-Aldrich (St. Louis, MO, USA). Phosphate-buffered saline (PBS) and fetal bovine serum (FBS) were purchased from Thermo Fischer Scientific (Waltham, MA, USA).

Plant material and extraction procedure. Rheum officinale was collected between August and September of 2012 from a local site in Guangzhou, China. The identity of the plant material was confirmed by an experienced taxonomist. The rhizomes of Rheum officinale were thoroughly washed with tap water, shade dried and cut into small sections. Ethanol (95\%) was used for hot extraction, which was conducted over $3 \mathrm{~h}$ using a soxhlet extraction apparatus (Jianhu Shendi Glass Instruments Co., Ltd., Yancheng, China). The extract was then concentrated under reduced pressure in a rotary evaporator (Hangzhou Greatcool Refrigeration Equipment Co., Ltd., Hangzhou, China) at $40^{\circ} \mathrm{C}$ and maintained in a refrigerator at $4^{\circ} \mathrm{C}$ prior to use. Emodin and aloemodin were obtained from Sigma-Aldrich.

Animals. Female Sprague-Dawley rats were obtained from the Experimental Animal Centre of Sichuan University (Chengdu, China). Animals were treated in accordance with the Guide for Animal Care and Use of Laboratory Animals (National Institute of Health, 1996). All procedures were approved by the Ethics Committee of the General Hospital of Chengdu Military Region (Chengdu, China).

Primary neuronal cultures. Primary neuronal cultures were obtained from the cortex of rat embryos aged 20 days. Pregnant rats were sacrificed by decapitation and the embryos were removed aseptically. Cortices were removed from the embryos using a dissection microscope (Ningbo Zhanjing Optical Instruments Co., Ltd., Ningbo, China). Tissues were ground and trypsinized using $0.21 \%$ trypsin-EDTA in $0.2 \mathrm{M}$ PBS for $10 \mathrm{~min}$ at $37^{\circ} \mathrm{C}$. Following centrifugation, tissues were suspended in modified Eagle's medium (MEM; Hangzhou Sijiqing Biological Products Co., Ltd., Hangzhou, China) containing $10 \%$ FBS and triturated with a Pasteur pipette (Runlab Labware Manufacturing Co., Ltd., Taizhou, China). The resulting single-cell suspension was collected and the cell density of the suspension was measured using a hemocytometer (BD Biosciences, Franklin Lakes, NJ, USA). Cells were cultured onto poly-D-lysine coated 96 -well plates $\left(1 \times 10^{6}\right.$ cells/well). Cell cultures were maintained at $37^{\circ} \mathrm{C}$ in a $\mathrm{CO}_{2}$ incubator. The resultant culture comprised $95 \%$ neuronal cells, $3.5 \%$ astrocytes, $0.5 \%$ oligodendrocytes, $0.6 \%$ microglia and $0.4 \%$ ependymal cells.

Exposure of primary cultures to ionizing radiation. Following culture for seven days, cells were treated with $20 \mu \mathrm{g} / \mathrm{ml}$ rhubarb extract, $10 \mu \mathrm{M}$ emodin and $10 \mu \mathrm{M}$ aloemodin in MEM and $10 \%$ FBS. The primary cultures were then subjected to irradiation in a ${ }^{137} \mathrm{Cs}$ irradiator (J.L. Shepherd and Associates, San Fernando, CA, USA) with a 2 Gy $\gamma$-ray dose. Following irradiation, cultures were maintained for $24 \mathrm{~h}$ at $37^{\circ} \mathrm{C}$ in a $7.5 \% \mathrm{CO}_{2}$ incubator prior to analysis.

Measurement of ROS generation. ROS generation was measured using a 2',7'-dichlorofluorescein (DCFH-DA) probe as described previously (18). DCFH-DA enters cells where it is cleaved by cellular esterases and becomes impermeable. The probe becomes fluorescent on oxidization by ROS. Cell cultures were washed with PBS, supplemented with $0.15 \mathrm{~g} / 1$ $\mathrm{CaCl}_{2}$ and $0.2 \mathrm{~g} / 1 \mathrm{MgCl}_{2}$, incubated with $10 \mu \mathrm{M}$ DCFH-DA for $30 \mathrm{~min}$, washed with PBS to remove any excess probe and incubated with $10 \mu \mathrm{g} / \mathrm{ml}$ rhubarb extract. After $3 \mathrm{~h}$, the cells were irradiated in a ${ }^{137} \mathrm{Cs}$ irradiator with a single dose of 2 Gy $\gamma$-rays. At $1 \mathrm{~h}$ following irradiation, ROS generation was measured using a FACS BD Calibur (BD Biosciences, Bedford, MA, USA), and BD Cell Quest Pro 6.0 software was used to analyze the data.

Lactate dehydrogenase ( $L D H)$ assay. At 24 h post-irradiation, the cell culture medium was harvested and rendered cell-free using centrifugation $(15,000 \mathrm{x} \mathrm{g}$ for $5 \mathrm{~min}$ at $4^{\circ} \mathrm{C}$ ). LDH Cytotoxicity Assay kit (Roche Diagnostics Corporation, Indianapolis, IN, USA) was used to measure the release of LDH from the cells. After a 40 min incubation at room temperature using the $\mathrm{LDH}$ kit, the $\mathrm{LDH}$ release was measured at a wavelength of $495 \mathrm{~nm}$ using a microplate system enzyme-linked immunospot assay reader (MHM-96B; Changchun MH Medical Co., Ltd., Changchun, 


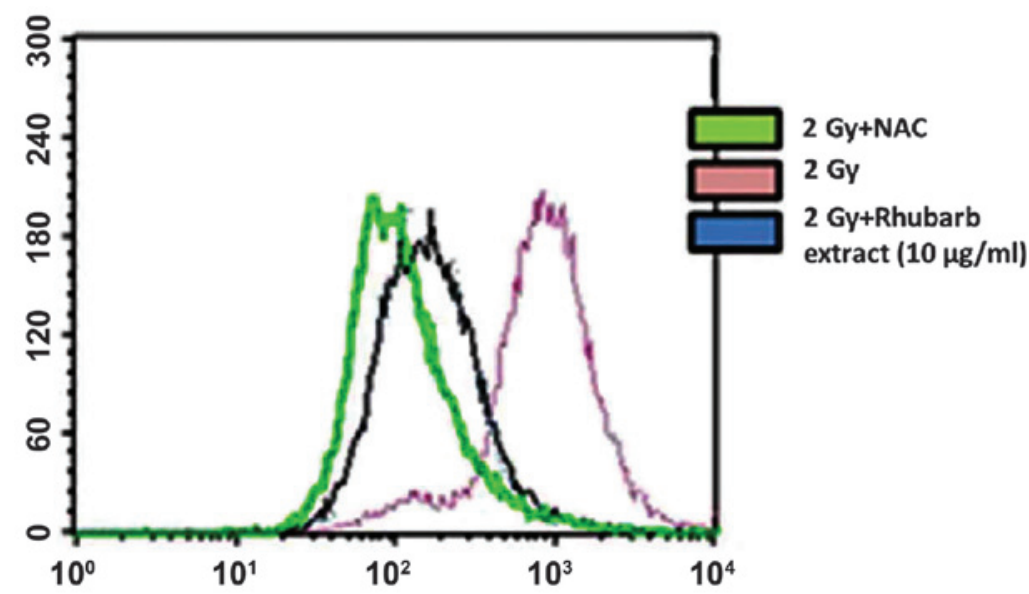

Figure 1. Radiation-induced ROS generation. Cells were pretreated with $10 \mu \mathrm{g} / \mathrm{ml}$ rhubarb extract, NAC or control for $3 \mathrm{~h}$. Cells were irradiated with a single dose of $2 \mathrm{~Gy} \gamma$-rays and ROS generation was measured $1 \mathrm{~h}$ post-irradiation. The results are presented as arbitrary fluorescence units. ROS, reactive oxygen species; NAC, $\mathrm{N}$-acetylcysteine.

China). Three different concentrations $(2,5$ and $10 \mu \mathrm{g} / \mathrm{ml})$ of the rhubarb extract were used to evaluate its effect on lactate dehydrogenase release in neuronal cell cultures.

Measurement of apoptosis. Bis-benzimide nuclear staining was used to detect DNA fragmentation. Cells were plated onto 96-well plates coated with poly-D-lysine for bis-benzimide staining. Cells were fixed with $5 \%$ paraformaldehyde in $0.1 \mathrm{M}$ PBS, washed with $0.1 \mathrm{mM}$ PBS and stained with bis-benzimide $(10 \mu \mathrm{g} / \mathrm{ml})$, which is a fluorescent DNA-binding dye, for $10 \mathrm{~min}$ at $25^{\circ} \mathrm{C}$. Three different concentrations $(2,5$ and $10 \mu \mathrm{g} / \mathrm{ml}$ ) of the rhubarb extract were used to investigate its effect on neuronal apoptosis. Cells were examined under an optical fluorescence microscope (Ningbo Sunny Instruments Co. Ltd., Zhejiang, China). The number of cells with apoptotic bodies per total cell number was calculated from 8-10 random fields of $6 \times 10^{3}$ cells/well. Three wells were assessed per treatment.

LC-ESI-MS-MS/HPLC analysis. LC-MS equipment consisted of a chromatographic system (LC-MS QqQ-6410B Agilent, 1260 Infinity series (Agilent Technologies, Santa Clara, CA, USA) coupled with an Agilent Triple Quad mass spectrometer fitted with an electrospray ionization source, using the following MS conditions: MS range, 100-1,200 Da; MS spectra obtained using positive and negative modes; nebulizer gas, $45 \psi$; and capillary voltage, $4000 \mathrm{~V}$.

HPLC analysis was conducted using an Agilent 1260 infinity series (Agilent Technologies). A chromolith RP-18e column (4.6 mm ID, $50 \mathrm{~mm}$ length) was used. The mobile phase consisted of (A) aqueous acetic acid (0.5\%) and (B) $70 \%$ methanol. The mobile phase gradient was as follows: 0-8 min, linear gradient from 10 to $25 \%$ of $\mathrm{B} ; 8-12 \mathrm{~min}$, isocratic conditions at $25 \%$ of $\mathrm{B} ; 12-16 \mathrm{~min}$, linear gradient from 25 to $40 \%$ of $\mathrm{B}$; $16-40 \mathrm{~min}$, linear gradient from 40 to $50 \%$ of B; and $40-50 \mathrm{~min}$, linear gradient from 50 to $100 \%$ of B. The flow rate used was $1 \mathrm{ml} / \mathrm{min}$.

Statistical analysis. All data are expressed as the mean \pm standard error of the mean. One way analysis of variance was used. All statistical analyses were conducted using SPSS version 11.5 (SPSS Inc., Chicago, IL, USA). P $\leq 0.05$ was considered to indicate a statistically significant difference.

\section{Results}

ROS generation. The present study used the oxidation-sensitive probe DCFH-DA to determine ROS generation in neuronal cells that had been incubated with the rhubarb extract prior to irradiation with a single dose of 2 Gy $\gamma$-rays. As hypothesized, irradiating the cells resulted in enhanced ROS production even at $1 \mathrm{~h}$ post-irradiation. This increased ROS generation was inhibited in cells treated with rhubarb extract at a dose of $10 \mu \mathrm{g} / \mathrm{ml}$. This result was confirmed by incubating the cells with $\mathrm{N}$-acetylcysteine, a known ROS scavenger. This also inhibited the radiation-induced increase in DCF fluorescence, which corresponded to decreased ROS generation (Fig. 1).

Cell apoptosis and lactate dehydrogenase release. Significant cell death, as measured by lactate dehydrogenase release, was observed following irradiation at a dose of 2 Gy. Irradiation reduced the numbers of neuronal cells by $50-55 \%$ and apoptosis was observed at $24 \mathrm{~h}$ post-irradiation. Using bis-benzimide staining, condensed and fragmented DNA was detected in the apoptotic cells. The extract exhibited a dose-dependent inhibition of radiation-induced apoptotic neuronal cell death, with the $10 \mu \mathrm{g} / \mathrm{ml}$ dose of the extract resulting in the greatest degree of inhibition ( $\mathrm{P}<0.05$; Figs. 2 and 3 ). Furthermore, in the bis-benzimide stained cultures, the rhubarb extract significantly reduced the number of neurons with condensed and fragmented DNA, particularly at the higher concentration $(10 \mu \mathrm{g} / \mathrm{ml})$. As shown in Fig. 2, healthy surviving neurons have a large, round and intact nucleus without any deformation, whereas in apoptotic neurons the chromatin is condensed and fragmented. As shown in Fig. 2, in the control cell cultures no apoptotic neurons were observed. In the cell cultures treated with rhubarb extract, few apoptotic neurons were visible. Administration of the extract also prevented the increase in lactate dehydrogenase release induced by irradiation $(\mathrm{P}<0.05)$. 

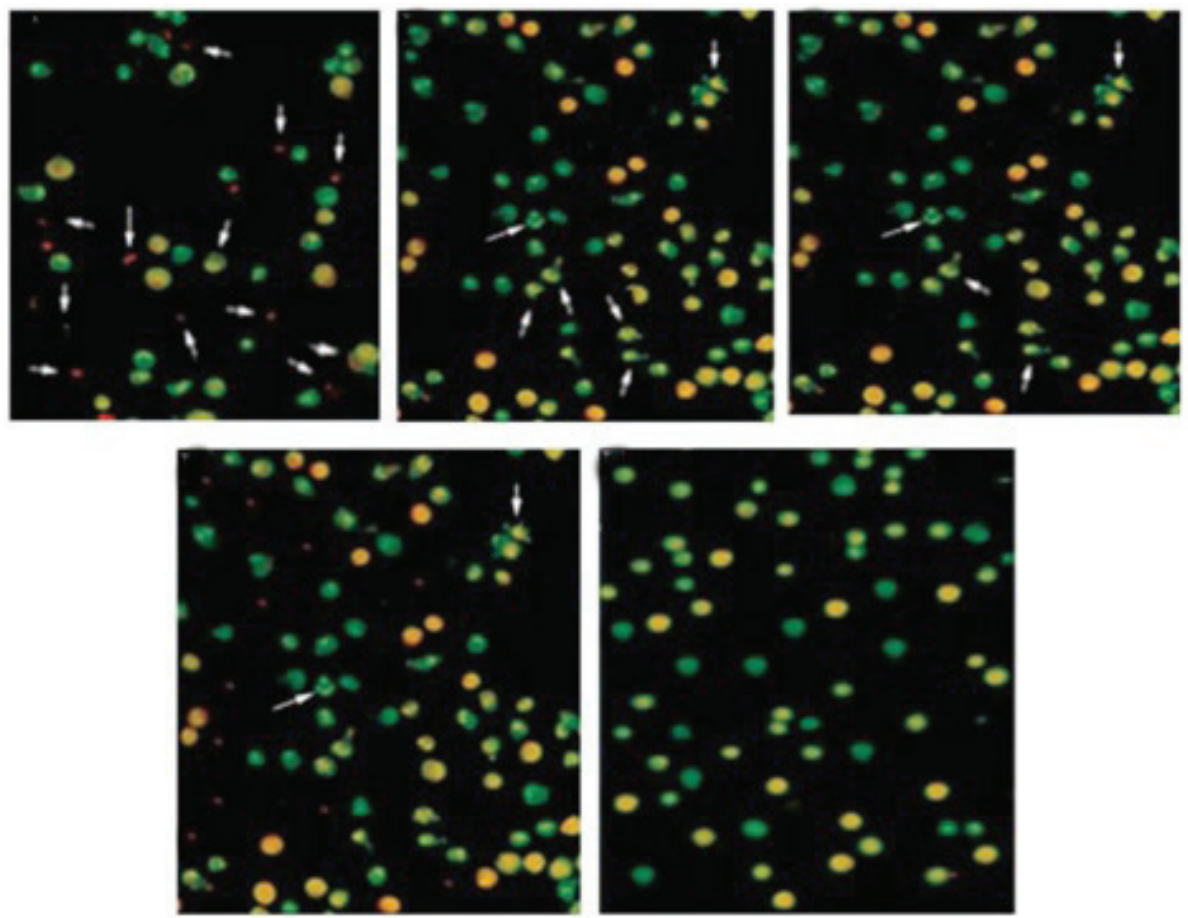

Figure 2. Fluorescent micrographs (using bis-benzimide staining) showing apoptotic fragmentation $24 \mathrm{~h}$ after $2 \mathrm{~Gy} \gamma$-ray irradiation. Bis-benzimide nuclear staining was used to detect DNA fragmentation. Healthy neuronal cells have a large, round and intact nucleus, whereas apoptotic neurons have condensed nuclei containing fragmented chromatin. Fragmented orange nuclei indicate apoptosis and green staining indicates intact nuclei. Magnification, x100.

A

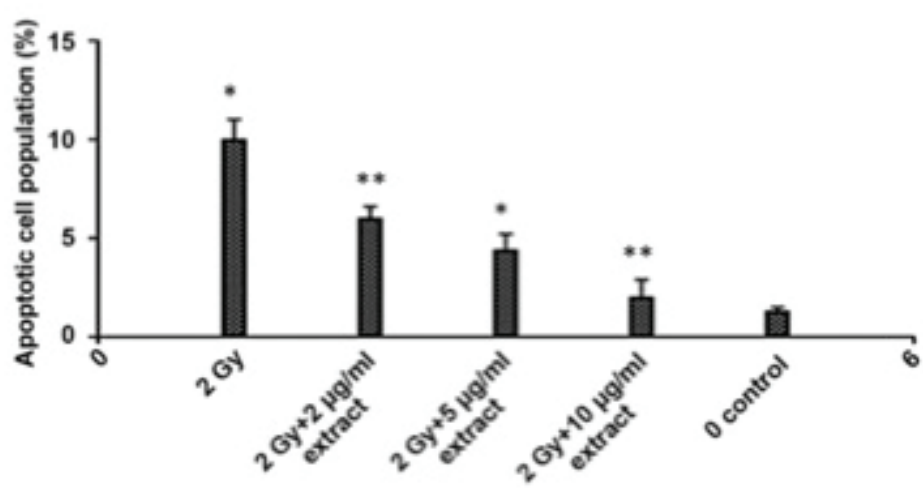

B

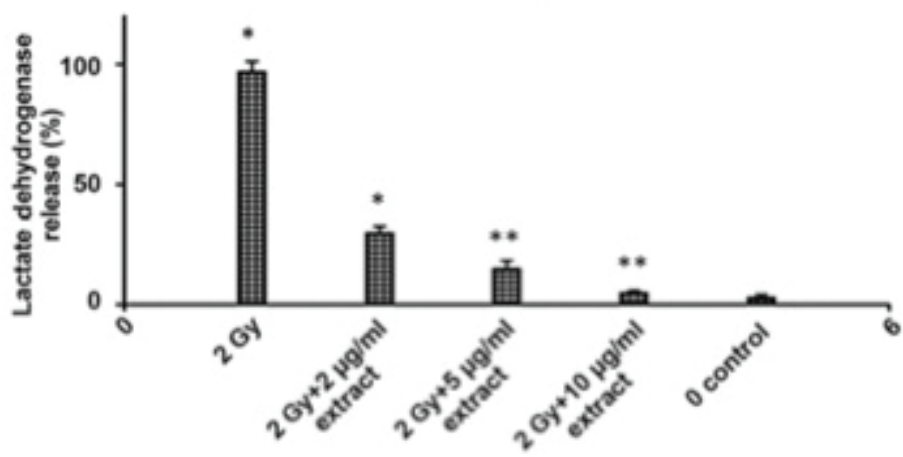

Figure 3. (A) Percentage of apoptotic neuronal cells still attached after $24 \mathrm{~h} 2 \mathrm{~Gy}$ irradiation, with and without rhubarb extract treatment, as detected by bis-benzimide staining. The extract exhibited a dose-dependent inhibition of apoptotic neuronal cell death, with $10 \mu \mathrm{g} / \mathrm{ml}$ extract showing maximum inhibition $(\mathrm{P}<0.05)$. (B) Lactate dehydrogenase release $24 \mathrm{~h}$ post $2 \mathrm{~Gy}$ irradiation, with and without rhubarb extract treatment. The extract exhibited a dose-dependent inhibition of lactate-dehydrogenase release with a maximum effect at a $10 \mu \mathrm{g} / \mathrm{ml}$ dose $(\mathrm{P}<0.05) * \mathrm{P}<0.05$, $* * \mathrm{P}<0.01$, as compared with the control cells.

Three different concentrations $(2,5$ and $10 \mu \mathrm{g} / \mathrm{ml})$ of the extract were used in the experiments that observed lactate dehydrogenase release and apoptosis. The extract exhibited a dose-dependent inhibition of lactate dehydrogenase release. The $10 \mu \mathrm{g} / \mathrm{ml}$ dose inhibited lactate dehydrogenase release by $>85 \%$ (Fig. 4). 
<smiles>CC1=CC2C(=O)C3=CC=CC(O)C3C(=O)C2C(O)=C1</smiles>

Chrysophanol<smiles>O=C1C2=CC=CC(O)C2C(=O)C2C(O)=CC(CO)=CC12</smiles>

Aloe-emodin<smiles>CC1=CC2C(=O)C3=CC(O)=CC(O)C3C(=O)C2C(O)=C1</smiles>

Emodin<smiles>COC1=CC(O)C2C(=O)C3C(O)=CC(C)=CC3C(=O)C2=C1</smiles>

Physcion<smiles>O=C(O)C1=CC2C(=O)C3=CC=CC(O)C3C(=O)C2C(O)=C1</smiles>

Rhein

Figure 4. Molecular structures of the polyphenolic compounds identified in the ethanol extract of Rheum officinale by liquid chromatography-mass spectrometry.

LC-MS-MS/HPLC analysis. The phytochemical analysis of the Rheum officinale extract was conducted by LC-ESI-MS and HPLC with diode-array detection techniques. The extract was run under positive and negative ESI-MS conditions and it showed several major and minor ionic species. Fragmentation of the major peaks was used for the identification of compounds present in the extract. The identification of the chemical compounds was also conducted by comparing the molecular ion peaks, along with the MS fragmentation patterns, with those in the literature. The five chemical constituents identified were emodin, aloe-emodin, chrysophanol, physcion and rhein (Fig. 4). Extraction of these phytoconstituents has previously been reported from this and other species of Rheum. These constituents have also been reported to possess a spectrum of biological properties, including antioxidant, antitumor and anti-inflammatory effects.

\section{Discussion}

The current study demonstrated that rhubarb extract provided significant protection against radiation-induced apoptosis and reduced ROS generation in primary neuronal cultures. These results suggest that rhubarb extract may be useful as a medicinal agent against radiation-induced neuronal apoptosis, which leads to cognitive impairment. The extract may also be of use in alleviating the oxidative stress that is induced following partial or whole brain irradiation. Oxidative stress has been reported to induce neuroinflammation, which is hypothesized to be involved in the development of radiation-induced brain injury.

Plant extracts contain a range of phytochemicals and therefore the radioprotective effects of these compounds are likely to be mediated through a number of mechanisms. Polyphenols in plants are involved in scavenging radiation-induced free radicals, in particular ROS which lead to DNA damage, such as single or double-strand breaks, base damage and DNA-DNA or DNA-protein cross-links. The DNA double strand breaks are hypothesized to be the most damaging events caused by ionizing radiation and are the primary mechanism leading to cell death due to irradiation. Polyphenols may also upregulate mRNA expression of antioxidant enzymes, including glutathione peroxidase, glutathione transferase, peroxidase, catalase and superoxide dismutase, and thus may alleviate the oxidative stress induced by ionizing radiation. The predominant medicinal constituents of rhubarb are emodin and aloemodin, which are polyphenols. Emodin has been reported to exhibit anti-inflammatory effects in a number of experimental models and any molecules with a similar structure are hypothesized to augment cancer therapy. The anti-inflammatory action of emodin is linked to its inhibition of nitric oxide and cytokine production. It may also inhibit superoxide production (19-21). Emodin or emodin-containing extracts, such as rhubarb, are also reported to promote an antioxidant status due to inhibition of free radical formation, free radical scavenging, 
inhibition of lipid peroxidation and increases in antioxidant defenses (22-25). Emodin or emodin-containing extracts thus provide protection to cell constituents in the presence of oxidative stress, such as that induced by irradiation.

In conclusion, rhubarb extract significantly reduced apoptotic neuronal cell death and inhibited ROS generation following irradiation. As such, rhubarb extract may be amenable to development for use as a therapeutic agent in radiation-induced brain injury, which is a risk factor for a number of chronic brain disorders.

\section{Acknowledgements}

This study was supported by the China Postdoctoral Science Foundation grant (grant no. 2013M542232).

\section{References}

1. American Cancer Society. Cancer Facts and Figures 2006. American Cancer Society, Atlanta, GA, 2006.

2. Siegel R, Naishadham D and Jemal A: Cancer statistics, 2012. CA Cancer J Clin 62: 10-29, 2012.

3. Jemal A, Bray F, Center MM, et al: Global cancer statistics. CA Cancer J Clin 61: 69-90, 2011.

4. Greene-Schloesser D and Robbins ME: Radiation-induced cognitive impairment - from bench to bedside. Neuro Oncol 14 (Suppl 4): iv37-iv44, 2012.

5. Patel RR and Mehta MP: Targeted therapy for brain metastases: improving the therapeutic ratio. Clin Cancer Res 13: $1675-1683,2007$.

6. Lowe XR, Bhattacharya S, Marchetti F and Wyrobek AJ: Early brain response to low-dose radiation exposure involves molecular networks and pathways associated with cognitive functions, advanced aging and Alzheimer's disease. Radiat Res 171: 53-65, 2009.

7. Armstrong CL, Hunter JV, Ledakis GE, Cohen B, Tallent EM, Goldstein BH, Tochner Z, Lustig R, Judy KD, Pruitt A, Mollman JE, Stanczak EM, Jo MY, Than TL and Phillips P: Late cognitive and radiographic changes related to radiotherapy: initial prospective findings. Neurology 59: 40-48, 2002.

8. Shaw EG, Rosdhal R, D'Agostino RB Jr, Lovato J, Naughton MJ, Robbins ME and Rapp SR: Phase II study of donepezil in irradiated brain tumor patients: effect on cognitive function, mood, and quality of life. J Clin. Oncol 24: 1415-1420, 2006.

9. Jakubs K, Bonde S, Iosif RE, Ekdahl CT, Kokaia Z, Kokaia M and Lindvall O: Inflammation regulates functional integration of neurons born in adult brain. J Neurosci 28: 12477-12488, 2008 .
10. Biscaro B, Lindvall O, Tesco G, Ekdahl CT and Nitsch RM: Inhibition of microglial activation protects hippocampal neurogenesis and improves cognitive deficits in a transgenic mouse model for Alzheimer's disease. Neurodegener Dis 9: 187-198, 2012.

11. Ekdahl CT, Kokaia Z and Lindvall O: Brain inflammation and adult neurogenesis: the dual role of microglia. Neuroscience 158: 1021-1029, 2009.

12. Linnik MD, Zobrist RH and Hatfield MD: Evidence supporting a role for programmed cell death in focal cerebral ischemia in rats. Stroke 24: 2002-2009, 1993.

13. Cotman CW and Anderson AJ: A potential role for apoptosis in neurodegeneration and Alzheimer's disease. Mol Neurobiol 10: 19-45, 1995.

14. Jagetia GC: Radioprotective potential of plants and herbs against the effects of ionizing radiation. J Clin Biochem Nutr 40: 74-81, 2007.

15. Sato M, Maulik G, Bagchi D and Das DK: Myocardial protection by protykin, a novel extract of trans-resveratrol and emodin. Free Radic Res 32: 135-144, 2000.

16. Kuo YC, Tsai WJ, Meng HC, Chen WP, Yang LY and Lin CY: Immune responses in human mesangial cells regulated by emodin from Polygonum hypoleucum ohwi. Life Sci 68: 1271-1286, 2001.

17. Peigen X, Liyi H and Liwei W: Ethnopharmacologic study of Chinese rhubarb. J Ethnopharmacol 10: 275-293, 1984.

18. Smith PS, Zhao W, Spitz DR and Robbins ME: Inhibiting catalase activity sensitizes 36B10 rat glioma cells to oxidative stress. Free Radic Biol Med 42: 787-797, 2007.

19. Wang CC, Huang YJ, Chen LG, Lee LT and Yang LL: Inducible nitric oxide synthase inhibitors of Chinese herbs III. Rheum palmatum. Planta Med 68: 869-874, 2002.

20. Chen Y, Yang L and Lee TJ: Oroxylin A inhibition of lipopolysaccharide-induced iNOS and COX-2 gene expression via suppression of nuclear factor-kappaB activation. Biochem Pharmacol 59: 1445-1457, 2000.

21. Kuo YC, Meng HC and Tsai WJ: Regulation of cell proliferation, inflammatory cytokine production and calcium mobilization in primary human T lymphocytes by emodin from Polygonum hypoleucum ohwi. Inflamm Res 50: 73-82, 2001.

22. Huang SS, Yeh SF and Hong CY: Effect of anthraquinone derivatives on lipid peroxidation in rat heart mitochondria: structure-activity relationship. J Nat Prod 58: 1365-1371, 1995.

23. Choi JS, Chung HY, Jung HA, Park HJ and Yokozawa T: Comparative evaluation of antioxidant potential of alaternin (2-hydroxyemodin) and emodin. J Agric Food Chem 48: 6347-6351, 2000.

24. Chiu PY, Mak DH, Poon MK and Ko KM: In vivo antioxidant action of a lignan-enriched extract of Schisandra fruit and an anthraquinone-containing extract of Polygonum root in comparison with schisandrin B and emodin. Planta Med 68: 951-956, 2002.

25. Du Y and Ko KM: Effects of emodin treatment on mitochondrial ATP generation capacity and antioxidant components as well as susceptibility to ischemia-reperfusion injury in rat hearts: single versus multiple doses and gender difference. Life Sci 77: 2770-2782, 2005. 